\title{
Low Temperature Exciton-Exciton Annihilation in Amphi-PIPE J-Aggregates
}

\author{
C. Spitz ${ }^{1}$ and S. Daehne ${ }^{2}$ \\ ${ }^{1}$ Department of Physics, University of Potsdam, am Neuen Palais 10, 14469 Potsdam, Germany \\ ${ }^{2}$ BAM, Department of Analytical Chemistry, 12489 Berlin, Germany
}

Received 2 May 2006; Revised 23 August 2006; Accepted 28 August 2006

The mobility of optically excited excitons on J-aggregates can be demonstrated by the phenomena of exciton-exciton annihilation. In this intensity-dependent process the collision of two excitons results in their annihilation and hence in a shortening of the mean excitation lifetime. By measuring the intensity-dependent fluorescent lifetime in contrast to the predicted immobilization of the excitons at low temperature we could prove the excellent mobility of the excitons at a temperature $(4 \mathrm{~K})$, which is far below their expected freezing point.

Copyright (c) 2006 C. Spitz and S. Daehne. This is an open access article distributed under the Creative Commons Attribution License, which permits unrestricted use, distribution, and reproduction in any medium, provided the original work is properly cited.

\section{INTRODUCTION}

In highly ordered antenna systems of photosynthetic bacteria [1] the optically excited energy is effectively transported by excitonic coupled pigments. Therefore, such systems are promising candidates for light harvesting in technological applications. Inparticular dye aggregates provide a convenient and cheap system because of their ability of selforganizing. The so called J-aggregates that are characterized by an extremely narrow absorption band, which is red shifted in respect to the monomer absorption, have already proved their technical use in many applications such as spectral sensitization in silver halide photography and charge generation in xerography process (e.g., $[2,3])$. Moreover photolysis of water by solar energy has already been realized by the use of J-aggregates [4].

Recently, a new class of dye aggregates was found in case of self-assembling amphiphilic dye molecules, which exhibit an excitonic split absorption spectrum $[5,6]$ and spontaneous symmetry breaking while building up chiral structures [7-9]. The behavior of these aggregates under very high pressure indicates a hollow structure [10] that was proved by cryo transmission electron microscopy (cryo-TEM). Comparable to the very large 2D J-aggregates of cyanine dyes found by Tiddy et al. [11], their size reaches several micrometers in solution and they are favorable candidates for light harvesting systems. Owing to their large-scale energy transport ability for dyes forming this new type of aggregates, the new expression amphi-PIPEs (amphiphilic molecules with pigment interaction performing energy-migration) is suggested [12]. In dependence of the chemical structure and environmental conditions they appear as helical cylinders, one and more layered tubes or vesicles, or as bilayer ribbons [1315]. The structure is governed by a sensitive equilibrium between hydrophobic, hydrophilic, electrostatic, and dispersion interaction forces of their chromophores [15]. In this paper we concentrate on cylindrical aggregates of the amphiPIPEs 1 because their geometry allows to describe the excitonic coupling of the chromophores in an analytical way $[12,16]$.

In general, the excitation energy from visible optical excitation on J-aggregates is delocalized over many molecules as so called Frenckel excitons [17]. The delocalization length, which is defined as number of molecules taking part at the exciton at the same time, extends about 10 molecules at room temperature [18] and up to 50 to 100 molecules at low temperatures [19-22]. However, the number of molecules that simultaneously form the exciton state is much bigger. This is demonstrated by the very low number of quenching molecules that is necessary to drastically reduce the fluorescence quantum yield of J-aggregates [23]. This is usually interpreted as exciton migration over large distances. A further proof of the exciton migration is given by the observation of exciton-exciton annihilation (EEA) on J-aggregates [24-26]. EEA means that two excitons on the aggregate interact and form a higher excited state of a single molecule, which is 
thermally relaxing to the ground state or to a single exciton. This way one or both excitons are annihilated, which results on one hand in a shortening of the fluorescence lifetime and on the other hand in a decreasing fluorescence quantum yield. When the low temperature kinetics of exciton-exciton annihilation of weakly localized Frenckel excitons was investigated analytically and in simulations $[27,28]$, it turned out that the annihilation kinetics divides up into two components: on the one hand, the very fast kinetics (200 fs given by the lifetime of higher exciton states [22]) at high excitation densities, when two or more excitations are created on the same group of molecules by one laser pulse, and, on the other hand, a very slow component due to the annihilation of excitons that are created on separate segments on the aggregate. As the latter is much slower than the spontaneous relaxation, the authors in $[27,28]$ suggest that this intra-segment annihilation is not observable and can be neglected in experimental investigations. At intensities as low that practically no excitations are created on the same segment, the only mechanism for EEA is given by motion of the excitons along the aggregate to meet on the same segment. Hence the size of the domain on which the excitons are able to migrate can be estimated by the minimal density of excitons that is necessary to observe EEA. In this way the exciton migration was found on domains of $6 \times 10^{4}$ molecules at room temperature [26] and $6 \times 10^{6}$ molecules at $77 \mathrm{~K}$, respectively [29]. As the localization segments of lowest single exciton states normally do not overlap [30, 31], excitons on J-aggregates are assumed to be immobilized at low temperature and EEA can happen only with two excitons generated at the same segment. Consequently low annihilation rates at low temperatures were predicted [27, 28]. The excitons are immobilized when the thermal energy kT does not enable them to escape low energy sites that exist due to the disorder of the aggregate. In contradiction, the loss of fluorescence polarization of the amphi-PIPEs of dye 1 at $4 \mathrm{~K}$ gives rise to increasing mobility of the excitons at lower temperature [12]. Moreover a nonmonotonous behavior of the annihilation rate as a function of temperature was found by Scheblykin et al. [32, 33]. In the Davydov split aggregates of THIATS they found the maximum annihilation rate around $50 \mathrm{~K}$. These experimental results recently induced the reinterpretation of the theoretical description [34]. Herein a freezing point for EEA was estimated which is given by a characteristic energy called $\varepsilon_{12}$ in [34].

In view to the amphi-PIPEs investigated in this paper a first rough estimation for the freezing point can be derived from the absorption spectrum. As the line width of the J-band is a measure of the disorder of the aggregate, the immobilization temperature is given by the comparison of the temperature dependent line width of the J-band to the thermal energy kT. The line width of the J-band of dye 1 fits well to the power law [35] $\Delta v=70 \mathrm{~cm}^{-1}+(5.8 \times$ $\left.10^{-6}\right) \mathrm{cm}^{-1} \times(\mathrm{T} / \mathrm{K})^{2.8}$. Hence, below $100 \mathrm{~K}$ the thermal energy $\mathrm{kT}$ is smaller than the line width and therefore the excitons should be immobilized. Based on the actual theoretical work [34] $\varepsilon_{12}$ can be estimated either from the coherent

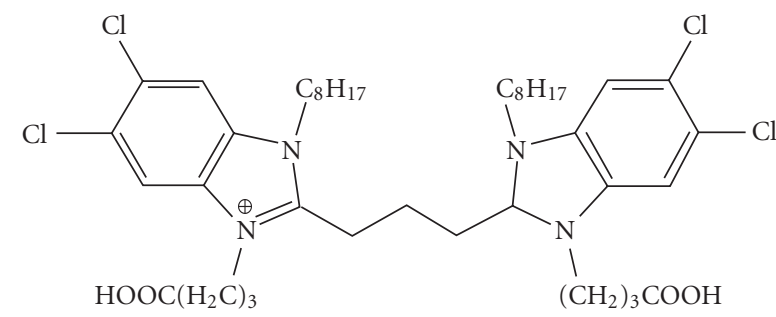

SCHEME 1

delocalization length or from the line width of the J-band. The former leads to a freezing point at $12 \mathrm{~K}$, the latter at $25 \mathrm{~K}$.

The aim of this paper is to prove the existence of exciton migration by EEA at low temperatures when, in the sense of common understanding, it should be forbidden. The exact quantification of the annihilation rate is still unclear and under discussion [36] and the values given in literature are hardly comparable. Therefore, we will not calculate explicit values of the migration rate as it was done in [37] for example. Instead we restrict ourselves in this paper to the proof of EEA on the one hand and to give the domain size of exciton migration on the other hand.

\section{EXPERIMENTAL}

Dye 1 (see Scheme 1) was purchased from FEW chemicals [38] and further purified as described in [12]. To prepare cylindrical J-aggregates for the low temperature measurements, dye 1 was solved $5 \times 10^{-3}$ molar at room temperature in a mixture of $20 \% \mathrm{~V} / \mathrm{V}$ ethylene glycol and $80 \% \mathrm{~V} / \mathrm{V}$ aqueous $10^{-2} \mathrm{M} \mathrm{NaOH}$. The solution has a triple split absorption spectrum with maxima at $573 \mathrm{~nm}, 595 \mathrm{~nm}$, and $603 \mathrm{~nm}$. In order to avoid crystallization and to yield an optically clear film, the solution was embedded between two $0.4 \mathrm{~mm}$ thick glass slides which were mounted in a copper sample holder and splashed into liquid nitrogen before cooling down to the aimed temperature of $4 \mathrm{~K}$ by $\mathrm{He}$ evaporation in an open cycle Oxford cryostat. The optical density was less than 1 in the maximum of absorption at $603 \mathrm{~nm}$. From this value the thickness of the film can be estimated to be less than $7.5 \mu \mathrm{m}$.

The fluorescence lifetime was measured by time correlated single photon counting technique (TCSPC) $[39,40]$ in dependence on the excitation intensity. A Rhodamine $6 \mathrm{G}$ dye laser synchronously pumped by an $80 \mathrm{MHz}$ mode locked argon ion laser provided pulses at $580 \mathrm{~nm}$ with 4 ps FWHM pulse duration. These pulses were used to excite the sample with a Gaussian beam profile of $1.6 \mathrm{~mm}$ FWHM. The fluorescence was selected by a subtractive double monochromator and detected by a fast Hamamatsu microchannel plate. For each detected fluorescence photon the delay time was electronically measured in respect to the excitation pulse. These delay times were accumulated over a large number of laser pulses to yield the fluorescence decay. 


\section{RESULTS}

The TCSPC principle allows to measure at very low excitation intensity where EEA can be excluded. The lowest excitation intensity that we used was $10^{-17} \mathrm{~J} \mathrm{pulse}^{-1}$ meaning that at each laser pulse less than 30 photons are absorbed. While increasing the excitation intensity the fluorescence was attenuated in front of the detector in order to keep the mean number of detected fluorescence photons per laser pulse less than $10^{-3}$. In order to avoid systematic error by sample degradation, the intensity was varied first increasing and then decreasing along the whole intensity scale. Within the experimental error no effect of degradation was observable.

The system response function of the setup was determined by measuring the scattered light of the laser beam. It is shown in Figure 1 together with selected fluorescence decays at $4 \mathrm{~K}$ and $600 \mathrm{~nm}$, differing only in excitation intensity. The width of the system response was 37 ps FWHM while the fluorescence had decay times of about 80 ps. In this condition we did not deconvolute the decay by the expected nonexponential multiparameter behavior of annihilation processes $[25,27,28,41-46]$. In order to quantify the intensity dependent shortening of the fluorescence decays the onset decay time was determined by deconvoluting the first $250 \mathrm{ps}$ of each decay with the system response assuming a single exponential decay. The resulting decay times are presented as squares in Figure 2 as a function of excitation intensity. The relative fluorescence quantum yield was determined by integrating the normalized decay up to $8 \mathrm{~ns}$ where all measured decays reached zero. The values are normalized and presented as open circles in Figure 2. It turns out that the decay time as well as the fluorescence quantum yield are independent of the excitation intensity up to $10^{3}$ photons/pulse and clearly are decreasing at higher photon rates. The mark of $20 \%$ fluorescence quenching is hit at $5 \times 10^{6}$ photons/pulse $\times \mathrm{cm}^{2}$ which is more than two orders of magnitude lower intensity as found in THIATS aggregates in the maximum of the annihilation rate around $50 \mathrm{~K}[32,33]$.

\section{DISCUSSION}

As the shortening of the fluorescence decay might be due to heating of the samples by the laser irradiation the influence of temperature was separately determined. Increasing the sample temperature from $5 \mathrm{~K}$ to $25 \mathrm{~K}$ causes a reduction of the fluorescence decay time from 80 to 60 ps. Even assuming $100 \%$ absorption and total conversion of the pulse energy in heat the warming up of the sample is less than $1 \mathrm{~K}$ at the highest intensity used in the experiment (see appendix). Therefore, it is unlikely that thermal effects govern the excitation dependence shown in Figure 2.

The calculation of the relative quantum yield by integrating the normalized decay curve is based on the assumption that the initial fluorescence intensity is a good measure for the total number of excitations generated. Without deconvoluting the system response the results can be distorted by processes that occur on the time scales relevant to the time res-

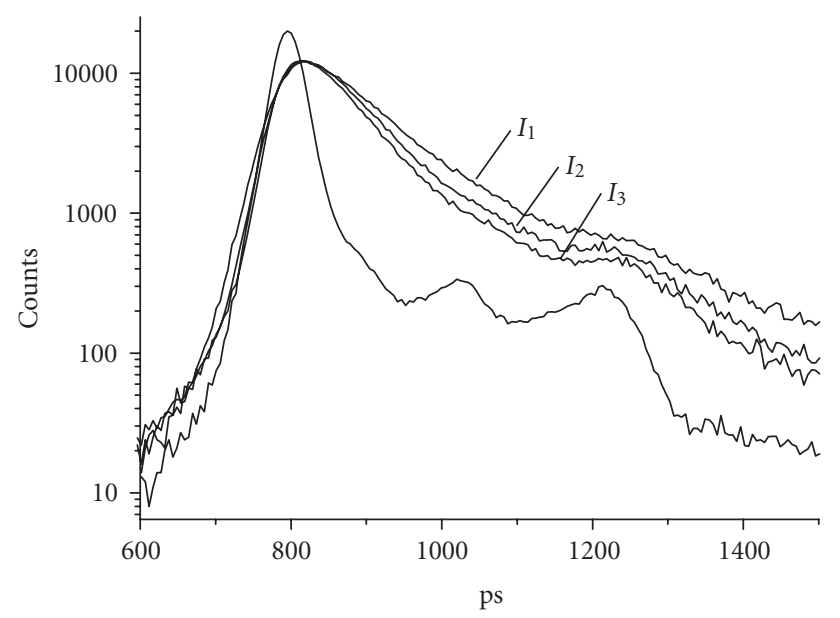

FIgURE 1: Fluorescence decays of the J-aggregates of dye 1 measured at $4 \mathrm{~K}$ and $600 \mathrm{~nm}$ after excitation at $580 \mathrm{~nm}$ with intensities $I_{1}=1.26 \times 10^{2}$ photons $/$ pulse, $I_{2}=2.70 \times 10^{4}$ photons $/$ pulse, and $I_{3}=1.17 \times 10^{5}$ photons/pulse. The 4 th decay is the system response measured at the excitation wavelength that was scattered by the sample.

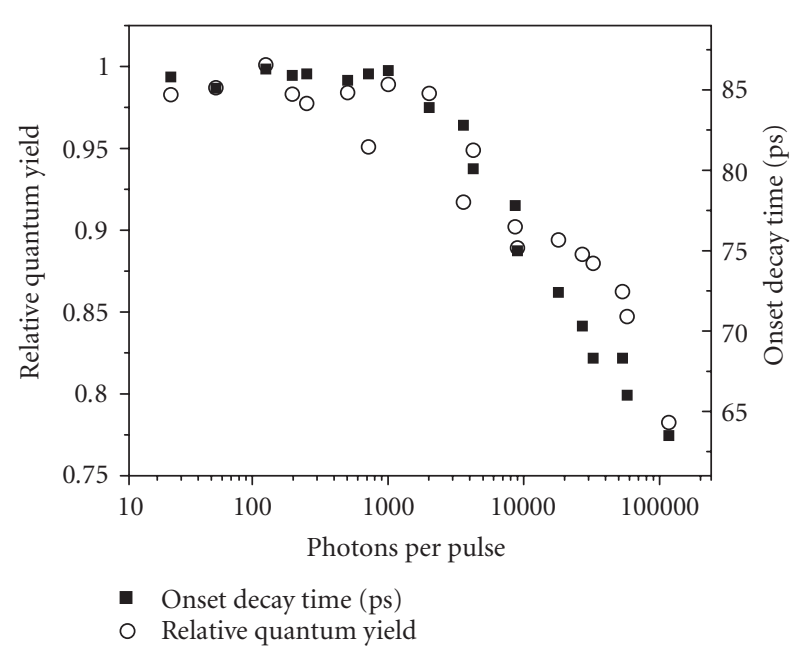

FIGURE 2: Squares: onset decay of the fluorescence of J-aggregates of dye 1 at $4 \mathrm{~K}$ in dependence on the excitation intensity determined by single-exponential deconvolution of the first 250 ps of each decay with the response function of the setup. Circles: relative fluorescence quantum yield, determined by integrating the normalized decay up to $8 \mathrm{~ns}$.

olution of the experiment. For this reason one must be careful when interpreting the exact development of the quantum yield given in Figure 2 as a function of excitation intensity. Nevertheless, without any doubt the quantum yield becomes intensity dependent at intensities higher than $10^{3}$ photons per pulse.

Even more caution is necessary in the interpretation of the decay times given in Figure 2 as there is no physical justification for characterizing the decay by taking into account 
only the first $250 \mathrm{ps}$ and deconvolute them as single exponential decay. But without committing to one explicit description of exciton-exciton annihilation it turned out that in our case the short time behavior of the decay was much stronger influenced by the excitation intensity than the long time components. Therefore the values have much less variation than the quantum yield data.

The data presented in Figure 2 suggests to follow the points as a straight line in the half logarithmic plot and extrapolate to a sharp bend at $10^{3}$ photons per pulse. But as the calculation of the values is not based on a concrete model this procedure is not physically justified. Therefore, within the experimental error one should consider an uncertainty of half an order of magnitude when interpreting this value. But there is no doubt that the measurements clearly prove effective exciton-exciton annihilation at low temperature of $4 \mathrm{~K}$.

\section{CONCLUSION}

At low temperature, in the common understanding, the thermal movement is very restricted and, therefore, optically excited localized Frenckel excitons are practically immobile $[27,28,34]$. The reason is that at temperatures with $\mathrm{kT}$ smaller than the difference between the local ground states, that is, the lowest exciton states on given segments of the aggregate, an exciton created in one of these states may move to another similar state only when the latter has an energy lower than the former. The typical energy offset between the local ground states can be estimated by numerical studies [34] and is of the order of the width of their energy distribution (i.e., the width of the J-band). Therefore, after one jump the exciton typically resides in the tail of this distribution. The number of states having still lower energy then drastically reduces, giving rise to a strong increase of the mean distance to such lower energy states. In fact, in one-dimensional aggregates already after one jump the exciton has a strongly suppressed chance to jump further $[27,28,30,31]$. Under our experimental conditions the spatial hopping of the excitons should be forbidden and excitonexciton annihilation could only occur at intensities where two excitations are created on the same segment $[27,28]$. In contradiction to this, we could proof in this paper an effective annihilation at low excitation intensities already at $4 \mathrm{~K}$.

Exciton-exciton annihilation in the amphi-PIPEs of dye $\mathbf{1}$ is starting at intensities higher than $10^{5}$ photons/pulse $\times \mathrm{cm}^{2}$, which corresponds to one single photon per $1000 \mu \mathrm{m}^{2}$ or one photon per $2 \times 10^{4}$ molecules, respectively. This is by orders of magnitude higher than the delocalization size $\mathrm{N}_{\text {del }}$, which covers 95 molecules in the case of cylindrical aggregates of this dye [47] and which varies between 50 and 100 molecules at other J-aggregates $[18,20]$. As the direct annihilation of two excitons, which are delocalized on different positions on the aggregate, can be neglected [27, 28], a lateral movement of the excitons even at very low temperatures covering their mean distance of $30 \mu \mathrm{m}$ within the lifetime of $85 \mathrm{ps}$ must be concluded. In difference to the investigations on THIATS ag- gregates $[32,33]$ in our case the considerable motion during the energy relaxation can be excluded because this runs on a subpicosecond time scale $[21,35]$. A motion over $30 \mu \mathrm{m}$ within femtoseconds would exceed velocity of light. In addition the recently observed fluorescence depolarization in the amphi-PIPEs at low temperature can only be explained under the consideration of an excellent mobility of the excitons at low temperatures [12]. As the hopping concept of exciton migration as given in [34] fails and moreover no dephasing of the excitations was found at low temperatures [47], a continuous coherent motion must be considered.

From the concentration of $3 \times 10^{21}$ molecules per liter, the irradiated sample volume of $1.5 \times 10^{-8}$ liter, and typically $10^{5}$ molecules per $\mu \mathrm{m}$ in double wall tubular aggregates of amphi-PIPE 1 [13], at the given excitation intensities one exciton has to jump over some thousands of aggregates before meeting an annihilation partner. Such unexpectedly high interaggregate transfer efficiency already was observed on the anomalous fluorescence depolarization at low temperature at these amphi-PIPEs.

In summary we found that at low temperature excitons move along amphi-PIPEs. The velocity can be estimated to $10^{5} \mathrm{~m} / \mathrm{s}$ by the distance of $30 \mu \mathrm{m}$ that is covered within the lifetime of 85 ps. It is interesting to note that this velocity means a dwell time of $10 \mathrm{fs}$ per molecule when moving along the aggregate, assuming that every step brings the exciton $1 \mathrm{~nm}$ further. This jump frequency is somewhat higher but within the same order of magnitude as it is to be expected by the dipole coupling of $1200 \mathrm{~cm}^{-1}$ [12]. The hereby-caused energy uncertainty covers the whole absorption band and therefore enables the exciton to escape the local traps.

This surprisingly high mobility may possibly be solved by considering the higher dimensionality of the tubular aggregates. It is known that the localization properties of quasi-particles, such as excitons, strongly depend on the dimensionality of the system. The one-dimensional case is the most extreme one, with exponential localization of all states. In two dimensions, the localization is much more subtle and often shows algebraic tails. In [48] exciton states in disordered cylindrical aggregates have been studied. Figure 5 of the cited paper shows autocorrelation plots of the wavefunctions at various energies, which (though not the focus of that paper) quite clearly demonstrate that the exciton states have quite long tails. Such long (nonexponential) tails in principle allow for contact between exciton states that are separated by more than the localization size as obtained from the commonly used participation ratio and may enhance the mobility even at low temperature. While this possibility has not been worked out in detail, the velocity and the range of the excitons seem to be still higher than expected.

A different aspect is recently given by a new theoretical approach: in contrast to the common understanding of the optical behavior as a function of disorder [12, 20, 21, 27, 28] the CES approximation $[49,50]$ predicts the whole spectral appearance as a function of vibrational states of the molecules. In this manner the line width is not any longer a criterion for disorder. Moreover from a narrow J-band 
one can conclude that the energy exchange between the molecules becomes faster than the vibrational modes. Therefore, from the narrowing of the J-band with decreasing temperature this theory predicts even higher mobility of the excitons at low temperature. Even though this theory cannot describe the results of holeburning or pump probe experiments or temperature-dependent absorption and fluorescence as was demonstrated for disordered systems in [51-53], the CES approach agrees with the high velocity of the excitons estimated from the results in this paper.

\section{APPENDIX}

Heating of the sample by laser irradiation estimated for the highest intensity used.

(i) Initial heating of the aggregates: mass of the irradiated aggregates $\sim 6 \times 10^{-11} \mathrm{~kg} \Rightarrow$ heat capacity (specific $\left.\sim 4200 \mathrm{~J} \mathrm{~kg}^{-1} \mathrm{~K}^{-1}\right) \sim 3 \times 10^{-3} \mathrm{~J} \mathrm{~K}^{-1}$ maximum pulse energy $4 \times 10^{-14} \mathrm{~J} \Rightarrow$ heating per pulse $=3 \times 10^{-8} \mathrm{~K}$.

As the aggregates are closely solved in the surrounding water (H-bonding to water causes aggregation) a good heat exchange is assumed leading to equilibration from pulse to pulse.

(ii) Heating of the sample volume by each single laser pulse: sample volume (diameter $1.6 \times 10^{-3} \mathrm{~m}$, height $7.5 \times$ $\left.10^{-6} \mathrm{~m}\right)=15 \times 10^{-12} \mathrm{~m}^{3} \Rightarrow$ sample mass $\sim 15 \times 10^{-9} \mathrm{~kg} \Rightarrow$ heat capacity $\sim 6 \times 10^{-5} \mathrm{~J} \mathrm{~K}^{-1}$ maximum pulse energy $4 \times$ $10^{-14} \mathrm{~J} \Rightarrow$ heating per pulse $=6 \times 10^{-10} \mathrm{~K}$ (at $80 \mathrm{MHz}$ pulse repetition the temperature would increase $2.8 \mathrm{~K} / \mathrm{min}$ without heat diffusion).

(iii) Vertical temperature gradient within the sample:

(a) heat diffusion from the sample to the glass plate: (area $A=2 \mathrm{~mm}^{2}$, laser power $p=3 \times 10^{-6} \mathrm{~W}$, length $d x=$ $7.5 \times 10^{-6} \mathrm{~m}$, temperature difference $d T$, heat diffusion coefficient $\left.\lambda \sim 2 \mathrm{~W} \mathrm{~m}^{-1} \mathrm{~K}^{-1}\right) p / A=\lambda d T / d x \Rightarrow$ temperature difference from sample to glass: $d T=10^{-5} \mathrm{~K}$,

(b) vertical heat diffusion within the glass plate: (analog, but $d x=4 \times 10^{-4} \mathrm{~m}$, factor 0.5 for diffusion to both glass plates $\Rightarrow$ vertical temperature gradient within the glass plate: $d T=3 \times 10^{-4} \mathrm{~K}$.

(iv) Radial heat diffusion to the copper holder: the laser power $p=3 \times 10^{-6} \mathrm{~W}$ must be transported through every circle $r$ around the sample (circumference $2 \pi r$, height $d=$ $0.8 \mathrm{~mm})$ by the heat current $j(r)=p /$ area $=p /(2 \pi r d)=$ $0.6 \mathrm{~W} \mathrm{~m}^{-1} / \mathrm{r}$.

As the radial heat current is given by $j(r)=-\lambda d T / d r$, the temperature must fulfill $d T / d r=-0.3 \mathrm{~K} / \mathrm{r}$ which is fulfilled by $T(r)=T_{0}-0.3 \mathrm{~K} \ln (r)$.

The copper holder $(r=5 \mathrm{~mm})$ is on $T(5 \mathrm{~mm})=4 \mathrm{~K}$.

Therefore the sample has the temperature $T(0.8 \mathrm{~mm})=$ $4 \mathrm{~K}+0.3 \mathrm{~K}(\ln (5)-\ln (0.8))=4.55 \mathrm{~K}$.

\section{ACKNOWLEDGMENTS}

This work has been supported by the Deutsche Forschungsgemeinschaft (SFB 337, AB 74/5-3). Very helpful discussions with Dr. V. A. Malyshev and Professor J. Knoester were made possible by the INTAS program no. 97-10434. We thank the authors of [48] for the interpretation of their work in respect to exciton mobility.

\section{REFERENCES}

[1] G. Mc Dermott, S. M. Prince, A. A. Freer, et al., "Crystal structure of an integral membrane light-harvesting complex from photosynthetic bacteria," Nature, vol. 374, pp. 517-521, 1995.

[2] G. Bach and S. Daehne, "Cyanine dyes and related compounds," in RODDs Chemistry of Carbon Compounds, 2nd suppl., M. Sainsbury, Ed., vol. IV of Heterocyclic Compounds, pp. 383-482, Elsevier Scienec, Amsterdam, The Netherlands, 2nd edition, 1997.

[3] P. M. Borsenberger and D. C. Hoesterey, "Hole photogeneration in aggregate photoreceptors," Journal of Applied Physics, vol. 51, no. 8, pp. 4248-4251, 1980.

[4] C. Köenigstein and R. Bauer, "Charge separation in Jaggregates of covalently linked cyanine dye-viologen systems," Solar Energy Materials and Solar Cells, vol. 31, no. 4, pp. 535539, 1994.

[5] U. De Rossi, J. Kriwanek, M. Lisk, et al., "Control of the J-aggregation phenomenon by variation of the N-alkylsubstituents," Journal fur praktische Chemie Chemiker-Zeitung, vol. 337, no. 1, pp. 203-208, 1995.

[6] A. Pawlik, S. Kirstein, U. De Rossi, and S. Daehne, "Structural conditions for spontaneous generation of optical activity in J-aggregates," The Journal of Physical Chemistry B, vol. 101, no. 29, pp. 5646-5651, 1997.

[7] C. Spitz, S. Daehne, A. Ouart, and H.-W. Abraham, "Proof of chirality of J-aggregates spontaneously and enantioselectively generated from achiral dyes," The Journal of Physical Chemistry $B$, vol. 104, no. 36, pp. 8664-8669, 2000.

[8] U. De Rossi, S. Daehne, S. C. Meskers, and H. P. Dekkers, "Spontane bildung von optischer Aktivität in J-aggregaten mit Davydov-aufspaltung," Angewandte Chemie, vol. 108, pp. 827830, 1996.

[9] U. De Rossi, S. Daehne, S. C. Meskers, and H. P. Dekkers, "Spontaneous formation of chirality in J-aggregates showing Davydov splitting," Angewandte Chemie International Edition in English, vol. 35, no. 7, pp. 760-763, 1996.

[10] C. Spitz and S. Daehne, "Architecture of J-aggregates studied by pressure-dependent absorption and fluorescence measurements," Berichte der Bunsen-Gesellschaft-Physical Chemistry, vol. 102, pp. 738-744, 1998.

[11] G. J. T. Tiddy, D. L. Mateer, A. P. Armerod, W. J. Harrision, and D. J. Edwards, "Highly ordered aggregates in dilute dye-water systems," Langmuir, vol. 11, no. 2, pp. 390-393, 1995.

[12] C. Spitz, J. Knoester, A. Ouart, and S. Daehne, "Polarized absorption and anomalous temperature dependence of fluorescence depolarization in cylindrical J-aggregates," Chemical Physics, vol. 275, no. 1-3, pp. 271-284, 2002.

[13] H. von Berlepsch, C. Böettcher, A. Ouart, C. Burger, S. Daehne, and S. Kirstein, "Supramolecular structures of Jaggregates of carbocyanine dyes in solution," The Journal of Physical Chemistry B, vol. 104, no. 22, pp. 5255-5262, 2000.

[14] H. von Berlepsch, C. Böettcher, A. Ouart, et al., "Surfactantinduced changes of morphology of J-aggregates: superhelixto-tubule transformation," Langmuir, vol. 16, no. 14, pp. 5908-5916, 2000.

[15] S. Kirstein, H. von Berlepsch, C. Böettcher, et al., "Chiral Jaggregates formed by achiral cyanine dyes," Chemistry / Physical Chemistry, vol. 1, no. 3, pp. 146-150, 2000. 
[16] M. Bednarz and J. Knoester, "The linear absorption and pump-probe spectra of cylindrical molecular aggregates," The Journal of Physical Chemistry B, vol. 105, no. 51, pp. 1291312923, 2001.

[17] A. S. Davydov, Theory of Molecular Excitons, Plenum Press, New York, NY, USA, 1971.

[18] M. van Burgel, D. A. Wiersma, and K. Duppen, "The dynamics of one-dimensional excitons in liquids," The Journal of Chemical Physics, vol. 102, no. 1, pp. 20-33, 1995.

[19] S. de Boer and D. A. Wiersma, "Dephasing-induced damping of superradiant emission in J-aggregates," Chemical Physics Letters, vol. 165, no. 1, pp. 45-53, 1990.

[20] J. R. Durrant, J. Knoester, and D. A. Wiersma, "Local energetic disorder in molecular aggregates probed by the one-exciton to two-exciton transition," Chemical Physics Letters, vol. 222, no. 5, pp. 450-456, 1994.

[21] J. Moll, S. Daehne, J. R. Durrant, and D. A. Wiersma, "Optical dynamics of excitons in J-aggregates of a carbocyanine dye," The Journal of Chemical Physics, vol. 102, no. 16, pp. 63626370, 1995.

[22] K. Minoshima, M. Taiji, K. Misawa, and T. Kobayashi, "Femtosecond nonlinear optical dynamics of excitons in Jaggregates," Chemical Physics Letters, vol. 218, no. 1-2, pp. 6772, 1994.

[23] G. Scheibe, A. Schoentag, and F. Katheder, "Fluoreszenz und Energiefortleitung bei reversibel polymerisierten Farbstoffen," Naturwissenschaften, vol. 27, no. 29, pp. 499-501, 1939.

[24] D. V. Brumbbaugh and A. A. Muenter, "Singlet exciton annihilation in the picosecond fluorescence decay of $1,1^{\prime}$ - diethyl2,2' -cyanine chloride dye J-aggregate," Journal of Luminescence, vol. 31-32, part 2, pp. 783-785, 1984.

[25] H. Stiel, S. Daehne, and K. Teuchner, "J-aggregates of pseudoisocyanine in solution: new data from nonlinear spectroscopy," Journal of luminescence, vol. 39, no. 6, pp. 351-357, 1988.

[26] V. Sundströem, T. Gillbro, R. A. Gadonas, and A. Piskarskas, "Annihilation of singlet excitons in J-aggregates of pseudoisocyanine (PIC) studied by pico- and subpicosecond spectroscopy," The Journal of Chemical Physics, vol. 89, no. 5, pp. 2754-2762, 1988.

[27] I. V. Ryzhov, G. G. Kozlov, V. A. Malyshev, and J. Knoester, "Low-temperature kinetics of exciton-exciton annihilation of weakly localized one-dimensional Frenkel excitons," The Journal of Chemical Physics, vol. 114, no. 12, pp. 5322-5329, 2001.

[28] V. A. Malyshev, G. G. Kozlov, H. Glaeske, and K.-H. Feller, "Channels of the exciton-exciton annihilation in onedimensional aggregates at low temperature," Chemical Physics, vol. 254, no. 1, pp. 31-38, 2000.

[29] I. G. Scheblykin, O. P. Varnavsky, M. M. Bataiev, O. Sliusarenko, M. Van der Auweraer, and A. G. Vitukhnovsky, "Non-coherent exciton migration in J-aggregates of the dye THIATS: exciton-exciton annihilation and fluorescence depolarization," Chemical Physics Letters, vol. 298, no. 4-6, pp. 341-350, 1998.

[30] V. A. Malyshev and P. Moreno, "Mirrorless optical bistability of linear molecular aggregates," Physical Review A, vol. 53, no. 1, pp. 416-423, 1996.

[31] V. A. Malyshev, H. Glaeske, and K.-H. Feller, "Excitonexciton annihilation in linear molecular aggregates at low temperature," Chemical Physics Letters, vol. 305, no. 1-2, pp. 117$122,1999$.
[32] I. G. Scheblykin, O. Yu. Sliusarenko, L. S. Lepnev, A. G. Vitukhnovsky, and M. Van der Auweraer, "Strong nonmonotonous temperature dependence of exciton migration rate in J-aggregates at temperatures from 5 to $300 \mathrm{~K}$," The Journal of Physical Chemistry B, vol. 104, no. 47, pp. 10949-10951, 2000.

[33] I. G. Scheblykin, O. Yu. Sliusarenko, L. S. Lepnev, A. G. Vitukhnovsky, and M. Van der Auweraer, "Excitons in molecular aggregates of 3,3'-Bis-[3-sulfopropyl]-5,5' -dichloro-9ethylthiacarbocyanine (THIATS): temperature dependent properties," The Journal of Physical Chemistry B, vol. 105, no. 20, pp. 4636-4646, 2001.

[34] A. V. Malyshev, V. A. Malyshev, and F. Domínguez-Adame, "Low-temperature quenching of one-dimensional localized Frenkel excitons," Chemical Physics Letters, vol. 371, no. 3-4, pp. 417-425, 2003.

[35] C. Spitz, Exzitonische anregungen in zylindrischen J-aggregaten von organischen Farbstoffen, Ph.D. thesis, Free University, Berlin, Germany, 1999, http://www.diss.fu-berlin.de/1999/15.

[36] J. Moll, W. J. Harrison, D. V. Brumbaugh, and A. A. Muenter, "Exciton annihilation in J-aggregates probed by femtosecond fluorescence upconversion," The Journal of Physical Chemistry A, vol. 104, no. 39, pp. 8847-8854, 2000.

[37] T. Kobayashi and S. Nagakura, "The biexcitonic quenching and exciton migration rate in aromatic crystals," Molecular Physics, vol. 24, no. 4, pp. 695-704, 1972.

[38] Dye 1 is available from FEW Chemicals, P. O. Box 1340, D06756 Wolfen, Germany.

[39] U. Resch and K. Rurack, "Steady-state and time-resolved fluorometry of fluorescent pollutants and heavy metal complexes," in Chemical, Biochemical and Environmental Fiber Sensors IX, vol. 3105 of Proceedings of SPIE, pp. 96-103, Munich, Germany, June 1997.

[40] M. Kollmannsberger, K. Rurack, U. Resch, and J. Daub, "Ultrafast charge transfer in amino-substituted boron dipyrromethene dyes and its inhibition by cation complexation: a new design concept for highly sensitive fluorescent probes," The Journal of Physical Chemistry A, vol. 102, no. 50, pp. 10211-10220, 1998.

[41] V. M. Kenkre, "Theory of exciton annihilation in molecular crystals," Physical Review B, vol. 22, no. 4, pp. 2089-2098, 1980.

[42] C. R. Doering and D. ben-Avraham, "Interparticle distribution functions and rate equations for diffusion-limited reactions," Physical Review A, vol. 38, no. 6, pp. 3035-3042, 1988.

[43] D. ben-Avraham, M. A. Burschka, and C. R. Doering, "Statics and dynamics of a diffusion-limited reaction: anomalous kinetics, nonequilibrium self-ordering, and a dynamic transition," Journal of Statistical Physics, vol. 60, no. 5-6, pp. 695728, 1990.

[44] R. Kopelman, A. L. Lin, and P. Argyrakis, "Non-classical kinetics and reactant segregation in $d$-dimensional tubular spaces," Physics Letters A, vol. 232, no. 1-2, pp. 34-40, 1977.

[45] R. Kopelman, S. Parus, and J. Prasad, "Fractal-like exciton kinetics in porous glasses, organic membranes, and filter papers," Physical Review Letters, vol. 56, no. 16, pp. 1742-1745, 1986.

[46] R. Kopelman, L. Li, S. Parus, and J. Prasad, "Exciton dynamics in thin wires," Journal of Luminescence, vol. 38, no. 1-6, pp. 289-294, 1987.

[47] S. S. Lampoura, C. Spitz, S. Daehne, J. Knoester, and K. Duppen, "The optical dynamics of excitons in cylindrical J-aggregates," The Journal of Physical Chemistry B, vol. 106, no. 12 , pp. 3103-3111, 2002. 
[48] C. Didraga and J. Knoester, "Optical spectra and localization of excitons in inhomogeneous helical cylindrical aggregates," The Journal of Chemical Physics, vol. 121, no. 21, pp. 1068710698, 2004.

[49] A. Eisfeld and J. S. Briggs, "The J-band of organic dyes: lineshape and coherence length," Chemical Physics, vol. 281, no. 1, pp. 61-70, 2002.

[50] A. Eisfeld and J. S. Briggs, "The J- and H-bands of organic dye aggregates," Chemical Physics, vol. 324, no. 2-3, pp. 376-384, 2006.

[51] M. Bednarz, V. A. Malyshev, and J. Knoester, "Temperature dependent fluorescence in disordered frenkel chains: interplay of equilibration and local band-edge level structure," Physical Review Letters, vol. 91, no. 21, Article ID 217401, 2003.

[52] D. J. Heijs, V. A. Malyshev, and J. Knoester, "Decoherence of excitons in multichromophore systems: thermal line broadening and destruction of superradiant emission," Physical Review Letters, vol. 95, no. 17, Article ID 177402, 2005.

[53] D. J. Heijs, V. A. Malyshev, and J. Knoester, "Thermal broadening of the $J$-band in disordered linear molecular aggregates: a theoretical study," Journal of Chemical Physics, vol. 123, no. 14, Article ID 144507, 12 pages, 2005. 


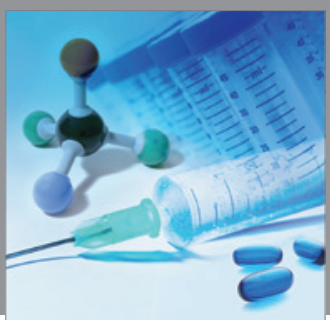

International Journal of

Medicinal Chemistry

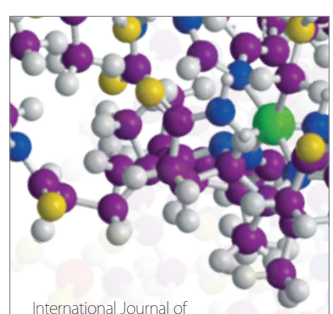

Carbohydrate Chemistry

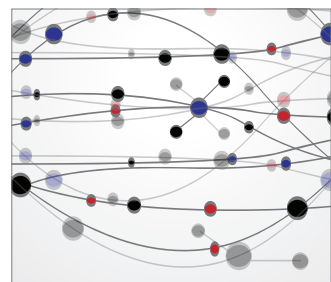

The Scientific World Journal
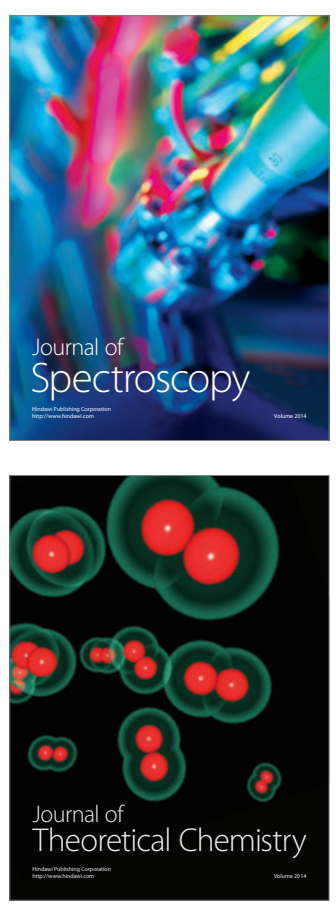
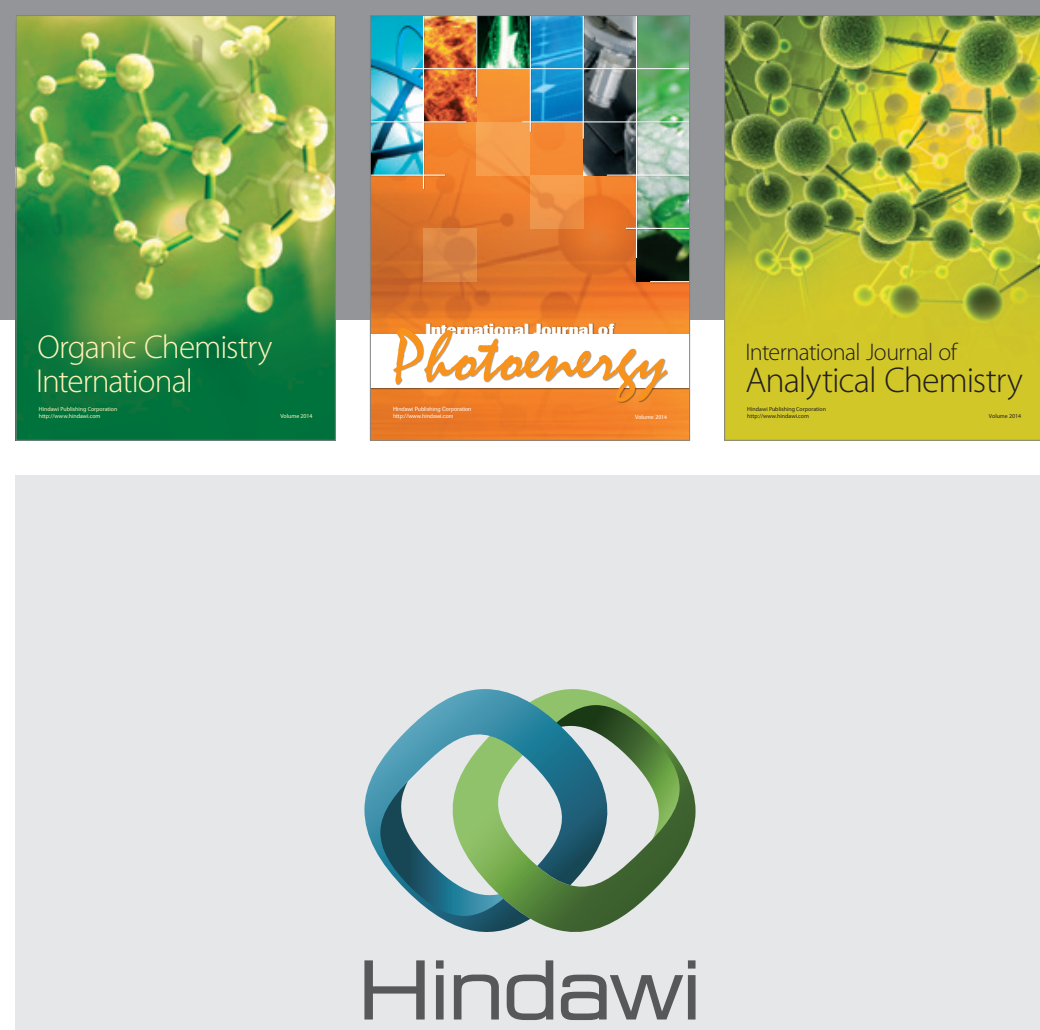

Submit your manuscripts at

http://www.hindawi.com
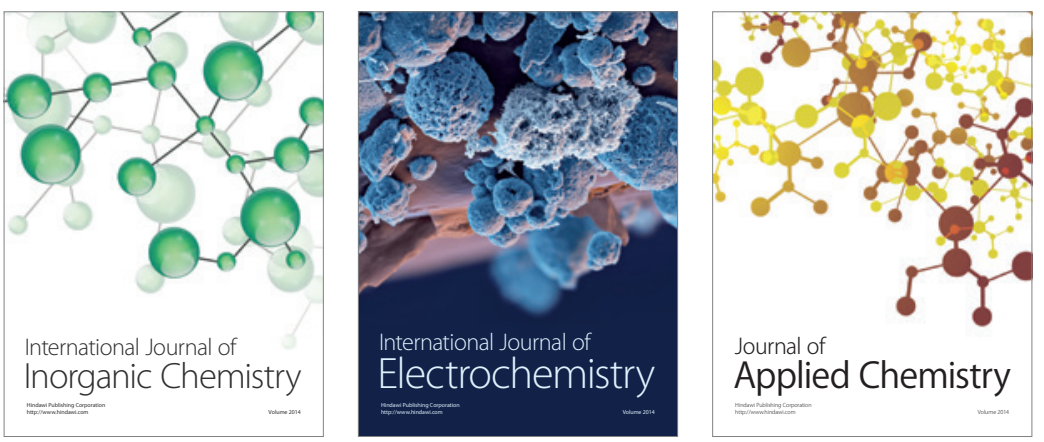

Journal of

Applied Chemistry
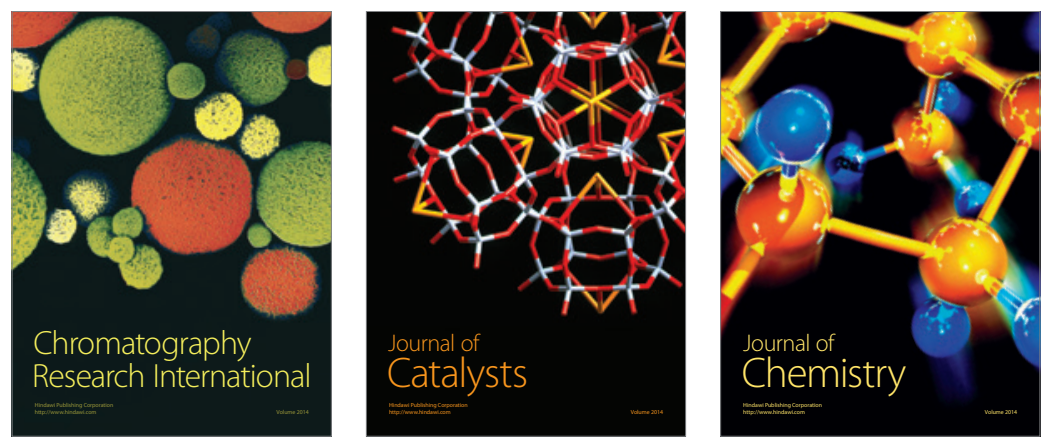
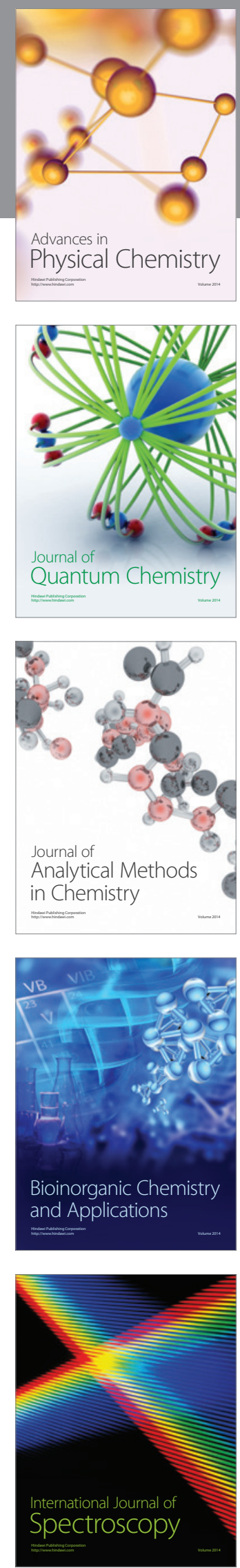